\title{
SISDLE: Sistema Inteligente de Suporte ao Descarte de Lixo Eletroquímico
}

\author{
Gil Eduardo de Andrade* \\ gil.andrade@ifpr.edu.br \\ Instituto Federal do Paraná
}

\author{
Diego Jonathan Hoss \\ diego.hoss@ifpr.edu.br \\ Instituto Federal do Paraná
}

\author{
Ana Paula Marques Barbosa \\ anapaullamb@gmail.com \\ Instituto Federal do Paraná
}

\author{
Fernanda Felisbino F. Silva \\ fernanda.felisbino@ufpr.br \\ Universidade Federal do Paraná
}

\begin{abstract}
Brazil has a prominent position in Latin America as the largest producer of toxic chemicals from battery disposal. These electrochemical components release toxic chemicals to the environment. In this context, the Smart System of Electrochemical Waste Disposal (SISDLE) proposes awareness about battery disposal. SISDLE is composed of a smart trash, which monitors the amount of discarded batteries, information sent to a database; a mobile app to inform where smart trash are located and provide the best route to them; and a web system that allows to monitor the fill rate for each smart trash and identify when collection is required.
\end{abstract}

\section{KEYWORDS}

Smart system, Discart, Electrochemical waste, IoT

\section{INTRODUÇÃO}

O Brasil possui posição de destaque na América Latina enquanto consumidor de produtos eletrônicos e produtor de resíduos. O primeiro mapa de lixo eletrônico mundial, coordenado pela Organização das Nações Unidas (ONU), apontou o Brasil como responsável pela produção de 1,4 milhão de toneladas lixo eletrônico em 2012, dentre as 49 milhões de toneladas produzidas mundialmente [2].

A demanda pela utilização de energia portátil é crescente em equipamentos eletroeletrônicos diversos, provocando o crescimento da produção e do consumo de pilhas e baterias [5]. Esses itens, ao serem descartados de modo inadequado, liberam componentes tóxicos ao ambiente, contaminando solo, água e atmosfera [1]. A Política Nacional de Resíduos Sólidos - PNRS (Lei n. ${ }^{\circ}$ 12.305/10) prevê que a responsabilidade sobre o produto cabe a comerciantes, fabricantes, importadores, distribuidores entre outros. Em outras palavras, a PNRS obriga que as empresas aceitem o retorno dos produtos descartados que por elas foram produzidos, sendo responsáveis também pela destinação dos mesmos, num processo denominado logística reversa [4].

Considerando essa problemática é propostro o Sistema Inteligente de Suporte ao Descarte de Lixo Eletroquímico SISDLE, que tem por objetivo auxiliar o processo de logistica reversa, promovendo a integração entre os agentes de descarte e as empresas responsáveis pelo recolhimento dos residuos.

\author{
Diego Stiehl \\ diego.stiehl@ifpr.edu.br \\ Instituto Federal do Paraná
}

\section{SOLUÇÃO PROPOSTA}

O SISDLE baseia-se no conceito de Cidades Inteligentes e IoT, visto que a solução busca promover o desenvolvimento urbano sustentável. Considerando a complexidade de experimentação inicial do SISDLE em ambientes urbanos, optou-se por projetá-lo para implementação no campus do Instituto Federal do Paraná - Campus Paranaguá. A escolha justificase porque o ambiente escolhido representa, em menor escala, o ambiente urbano e suas características.

A partir disso foram definidos dois requisitos principais para o SISDLE: (a) possibilitar que os usuários obtenham informações e rotas para lixeiras específicas para o descarte de pilhas/baterias; e (b) garantir que as empresas de coleta possam monitorar, em tempo real, a capacidade de armazenamento já utilizada de cada lixeira, sendo capazes de identificar o momento no qual o recolhimento das pilhas e baterias faz-se necessário.

Para contemplar as duas principais funcionalidades especificadas, para o ambiente escolhido, foram definidos: (a) os módulos que compõem o SISDLE; (b) a forma como se comunicam; e (c) seu modo de operação. O esquema contendo todos esses itens é apresentado na Figura 1.

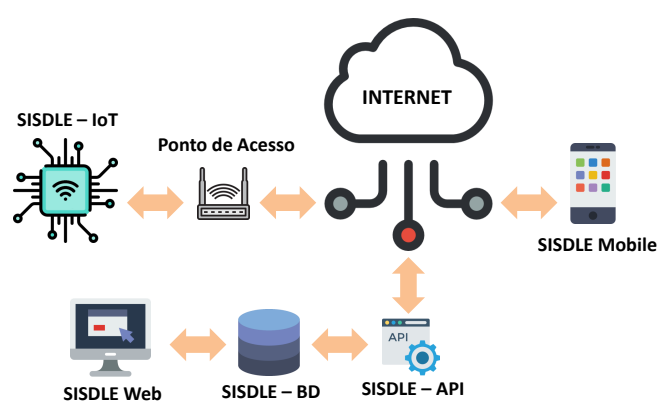

Figure 1: SISDLE - Módulos e Esquema de Funcionamento.

Nela é possível observar que o SISDLE é composto, basicamente, por três módulos: SISDLE IoT, SISDLE Mobile e SISDLE Web. Além deles, ainda fazem parte do seu modelo de funcionamento, como itens complementares, a API e o Banco de Dados SISDLE. Esses dois últimos são responsáveis, respectivamente, por dar acesso e armazenar as informações geradas e manipuladas pela aplicação. 
O módulo de IoT é o dispositivo de hardware projetado para tornar as lixeiras de coleta inteligentes, ou seja, capazes de monitorar a quantidade de pilhas e baterias descartas em seu recipiente, e transmitir essa informação via rede de internet sem fio. $\mathrm{O}$ m ódulo d e I oT f oi p rojetado para contemplar o requisito que permite, as empresas de coleta, monitorar e identificar o momento em que o recolhimento das pilhas e baterias é necessário. O SISDLE IoT é composto pelos seguintes dispositivos eletrônicos: placa NodeMCU ESP32, display LCD e sensores ultra-sônicos de distância, como pode ser visto no esquema da Figura 2.

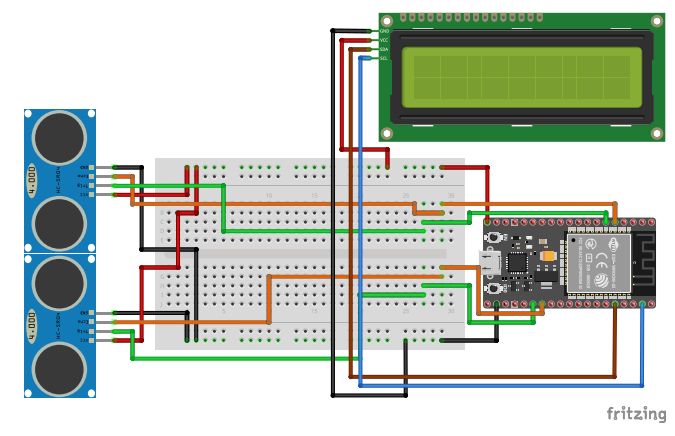

Figure 2: SISDLE IoT: Esquema de Funcionamento.

A placa NodeMCU ESP32 foi escolhida por ser uma plataforma de hardware de baixo custo e alto desempenho, que disponibiliza nativamente comunicação Wi-Fi e permite a criação de soluções de IoT [3]. A ESP é utilizada como placa mãe do SISDLE IoT, sendo programada para controlar os outros dispositivos eletrônicos, ao mesmo tempo em que está vinculada a um ponto de acesso, enviando e recebendo informações do SISDLE BD. Todo o tráfego de informações é efetuado, por questões de segurança e portabilidade, via requisições HTTPS à SISDLE API.

Os sensores ultra-sônicos são utilizados para medir a distância entre a sua localização (topo do recipiente) e o fundo da lixeira, como pode ser visto na Figura 3.

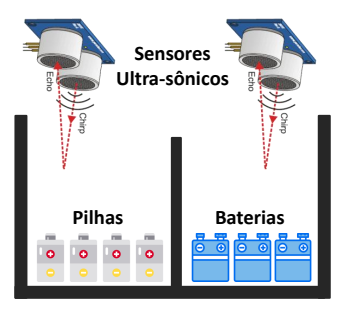

Figure 3: SISDLE IoT: Sensor Ultra-sônico de Distância.

De acordo com a distância medida é possível calcular o quanto (percentual) da capacidade do recipiente já foi preenchido por pilhas e baterias. São utilizados dois sensores porque a lixeira de descarte é composta por dois compartimentos, cada qual utilizado para armazenar um tipo de resíduo. Como os sensores são controlados pela ESP, essa informação é obtida pela placa e enviada via WiFi para o SISDLE BD.

Os módulos SISDLE Mobile e Web foram projetados para permitir, respectivamente, que os agentes de descarte obtenham informações e rotas sobre a localização das lixeiras inteligentes, e as empresas de coleta possam monitorar em tempo real o estado de armazenamento dos recepientes, sendo capazes de identificar o momento em que os resíduos devem ser recolhidos. O protótipo desses módulos pode ser visto na Figura 4.

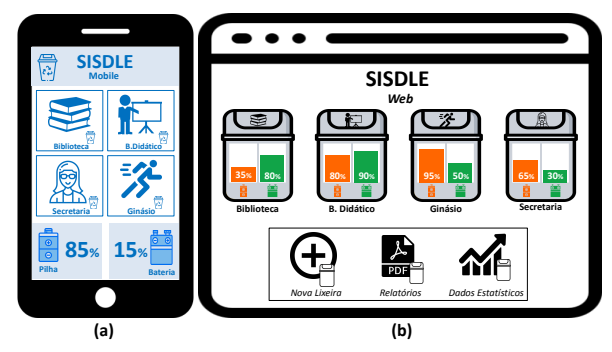

Figure 4: SISDLE IoT: Sensor Ultra-sônico de Distância.

\section{CONSIDERAÇÕES FINAIS}

Os próximos passos do projeto consistem na instalação do dispositivo de IoT nas lixeiras inteligentes e posterior implantação no campus. A partir disso serão coletados os dados que permitirão analisar o funcionamento do sistema, detectar e corrigir possíveis erros. Por fim, os módulos Web e Mobile serão adaptados para implantação no ambiente urbano, onde dois requisitos principais já foram definidos: (1) Web - utilização do mapa da cidade com disponibilização visual sobre a localização das lixeiras inteligentes; (2) Mobile - indicação das lixeira mais próxima com definição s obre a melhor rota de deslocamento até ela via API Google Maps ou similar.

\section{REFERENCES}

[1] J. C. Afonso, A. P. M. G. Barandas, G. A. P. Silva, and S. G. Fonseca. 2003. Processamento da pasta eletrolítica de pilhas usadas. In Quím. Nova, Vol. 26. São Paulo, SP.

[2] D. Brusamarelo, L. L. A. Pires, S. R. Longhin, A. R. Luz, and D. G. Miranda. 2016. A Educação Ambiental no Contexto da Evolução da Ciência, Tecnologia e Sociedade. In Revista HISTEDBR On-line. 336-346.

[3] M. Mehta. 2015. ESP 8266: A Breakthrough in Wireless Sensor Networks and Internet of Things. In International journal of Electronics and Communication Engineering Technology (IJECET), Vol. 6. 7-11.

[4] M. Morozesk and G. R. Coelho. 2016. Lixo Eletrônico "Uso e Descarte": uma proposta de intervenção em uma Escola Pública de Vitória-ES. In Revista Brasileira de Pesquisa em Educação em Ciências, Vol. 16. $317-338$.

[5] R. R. O. Rocha, Oliveira R. M., and T. G. S. Cruz. 2004. O descarte de pilhas e baterias no brasil e o seu impacto no meio ambiente. In Centro Superior de Educação Tecnológica-CESET, UNICAMP. 